\title{
EQUIVALENT CHARACTERIZATIONS OF HYPERBOLIC HÖLDER POTENTIAL FOR INTERVAL MAPS
}

\author{
HUAIBIN LI \\ (Communicated by Nimish Shah)
}

\begin{abstract}
Consider a topologically exact $C^{3}$ interval map without non-flat critical points. Following previous work we did, we give two equivalent characterizations of hyperbolic Hölder continuous potential in terms of the Lyapunov exponents and the measure-theoretic entropies of equilibrium states for those potentials.
\end{abstract}

\section{INTRODUCTION}

The thermodynamic formalism of smooth dynamical systems was initiated by Sinai, Ruelle, and Bowen [3,14]. For a uniformly hyperbolic diffeomorphism acting on a compact manifold of arbitrary dimension, they gave a complete description for Hölder continuous potentials. There have been several extensions of these results to one-dimensional maps that go beyond the uniformly hyperbolic setting. The lack of uniform hyperbolicity is usually compensated by an extra hypothesis on the potential. For example, there is a wealth of results for a piecewise monotone interval map $f: I \rightarrow I$ and a potential $\varphi$ of bounded variation satisfying $\sup _{I} \varphi<P(f, \varphi)$, where $P(f, \varphi)$ denotes the pressure; see for example [2, 4, 6, 8] and the references therein, as well as Baladi's book [1, §3]. Most results apply under the following weaker condition:

$$
\text { For some integer } \begin{aligned}
n \geq 1, \text { the function } S_{n}(\varphi):=\sum_{j=0}^{n-1} \varphi \circ f^{j} \text { satisfies } \\
\qquad \sup _{I} \frac{1}{n} S_{n}(\varphi)<P(f, \varphi) .
\end{aligned}
$$

In what follows, a potential $\varphi$ satisfying this condition is said to be hyperbolic for $f$.

In this paper, our goal is to give equivalent characterizations of hyperbolic Hölder continuous potentials for interval maps. In [7] the authors gave characterizations of hyperbolic Hölder continuous potentials for rational maps. Here we want to extend this result to interval maps in order to refer to them in the future. In order to state our main result, we briefly recall some concepts from thermodynamic formalism; see for example [11] for background.

Let $(X$, dist) be a compact metric space and $T: X \rightarrow X$ a continuous map. Denote by $\mathcal{M}(X)$ the space of Borel probability measures on $X$ endowed with the weak* topology, and by $\mathcal{M}(X, T)$ the subspace of $\mathcal{M}(X)$ of those measures that

Received by the editors November 5, 2013.

2010 Mathematics Subject Classification. Primary 37D35, 37E05.

Key words and phrases. Interval maps, hyperbolic Hölder continuous potentials, positive Lyapunov exponent.

The author was supported by the National Natural Science Foundation of China (Grant No. 11101124; and Grant No. 11471098). 
are invariant by $T$. For each measure $\mu$ in $\mathcal{M}(X, T)$, denote by $h_{\mu}(T)$ the measuretheoretic entropy of $\mu$. For a continuous function $\varphi: X \rightarrow \mathbb{R}$, denote by $P(T, \varphi)$ the topological pressure of $T$ for the potential $\varphi$, defined by

$$
P(T, \varphi):=\sup \left\{h_{\mu}(T)+\int_{X} \varphi d \mu: \mu \in \mathcal{M}(X, T)\right\} .
$$

A measure $\mu$ in $\mathcal{M}(X, T)$ is called an equilibrium state of $T$ for the potential $\varphi$ if the supremum in (1.1) is attained at $\mu$.

Given a compact interval $I$ of $\mathbb{R}$ and a differentiable map $f: I \rightarrow I$, a point of $I$ is critical if the derivative of $f$ vanishes at it. Denote by Crit $(f)$ the set of critical points of $f$. A differentiable interval map $f: I \rightarrow I$ is of class $C^{3}$ with non-flat critical points if it has a finite number of critical points and if:

- The map $f$ is of class $C^{3}$ outside $\operatorname{Crit}(f)$.

- For each critical point $c$ of $f$ there exist a number $\ell_{c}>1$ and diffeomorphisms $\phi$ and $\psi$ of $\mathbb{R}$ of class $C^{3}$, such that $\phi(c)=\psi(f(c))=0$, and such that on a neighborhood of $c$ on $I$, we have

$$
|\psi \circ f|=|\phi|^{\ell_{c}} .
$$

Throughout the rest of this paper, fix a compact interval $I$ of $\mathbb{R}$ and let $\mathscr{A}$ denote the collection of interval maps $f: I \rightarrow I$ of class $C^{3}$ with non-flat critical points. For an interval map $f$ of $\mathscr{A}$, denote by $|\cdot|$ the distance on $I$ induced by the norm distance on $\mathbb{R}$. Besides, for a subset $W$ of $I$ we use $|W|$ to denote the diameter of $W$ with respect to $|\cdot|$. For each measure $\mu$ in $\mathcal{M}(I, f)$, denote the Lyapunov exponent of $\mu$ by

$$
\chi_{\mu}(f):=\int_{I} \ln \left|f^{\prime}\right| d \mu .
$$

In what follows, we say that $f$ is topologically exact if for every open subset $U \subset I$ there is $n \geq 1$ such that $f^{n}(U)=I$. The main result of this paper is the following:

Theorem 1. Let $f: I \rightarrow I$ be an interval map in $\mathscr{A}$. If $f$ is topologically exact, then for every Hölder continuous potential $\varphi: I \rightarrow \mathbb{R}$, the following properties are equivalent:

1. The potential $\varphi$ is hyperbolic for $f$.

2. The measure-theoretic entropy of each equilibrium state of $f$ for the potential $\varphi$ is strictly positive.

3. The Lyapunov exponent of each equilibrium state of $f$ for the potential $\varphi$ is strictly positive.

Remark 1.1. The equivalence of properties 1 and 2 of Theorem 1 is part of Proposition 3.1 of [7, although we give the proof for the reader's convenience. On the other hand, recall that a map $f$ in $\mathscr{A}$ satisfies the Topological Collet-Eckmann Condition if there is a constant $\chi>0$ such that for every $\nu$ in $\mathcal{M}(I, f)$ we have $\int_{I} \ln \left|f^{\prime}\right| d \nu \geq \chi$; see 12 for other equivalent formulations. Let $f$ be a topologically exact map in $\mathscr{A}$ that satisfies the Topological Collet-Eckmann condition. Then every Hölder continuous potential is hyperbolic for $f$; see also [9] for another proof. 


\section{A REDUCTION}

In this section, we state first our main technical result as the "Key Lemma", whose proof occupies $\S \$ 3.1$ and 3.2 , and then we derive our Theorem 1 from it. In what follows, for each function $\varphi: I \rightarrow \mathbb{R}$ and each integer $n \geq 1$, put

$$
S_{n}(\varphi):=\varphi+\varphi \circ f+\cdots+\varphi \circ f^{n-1} .
$$

Key Lemma. Let $f$ be a map in $\mathscr{A}$ that is topologically exact and let $\nu$ be an invariant ergodic probability measure whose Lyapunov exponent is strictly positive. Then for every Hölder continuous function $\varphi: I \rightarrow \mathbb{R}$, there is a set of full measure of points $x_{0}$ such that

$$
\limsup _{n \rightarrow \infty} \frac{1}{n} \log \sum_{y \in f^{-n}\left(x_{0}\right)} \exp \left(S_{n}(\varphi)(y)\right)>\int_{I} \varphi d \nu .
$$

The following lemma is useful for the proof of Theorem 1 .

Lemma 2.1 (Lemma 2.8, 9]). Let $f$ be a map in $\mathscr{A}$ that is topologically exact, and let $\varphi: I \rightarrow \mathbb{R}$ be a continuous function. Then for every point $x_{0}$ of $I$, we have

$$
P(f, \varphi) \geq \limsup _{n \rightarrow \infty} \frac{1}{n} \log \sum_{y \in f^{-n}\left(x_{0}\right)} \exp \left(S_{n} \varphi(y)\right) .
$$

Proof of Theorem 1 assuming the Key Lemma. First, to prove the implication $1 \Rightarrow$ 2 , assume that $\varphi: I \rightarrow \mathbb{R}$ is a hyperbolic potential for $f$ and let $\nu$ be an equilibrium state of $f$ for the potential $\varphi$. Then there is an integer $n \geq 1$ such that $\sup _{I} \frac{1}{n} S_{n}(\varphi)<P(f, \varphi)$ and

$$
P(f, \varphi)=h_{\nu}(f)+\int_{I} \varphi d \nu
$$

Since $\nu$ is an invariant measure for $f$, we have

$$
\int_{I} S_{n}(\varphi) d \nu=\sum_{i=0}^{n-1} \int_{I} \varphi \circ f^{i} d \nu=n \int_{I} \varphi d \nu
$$

It follows that

$$
\begin{aligned}
h_{\nu}(f) & =P(f, \varphi)-\int_{I} \varphi d \nu=P(f, \varphi)-\frac{1}{n} \int_{I} S_{n}(\varphi) d \nu \\
& \geq P(f, \varphi)-\sup _{I} \frac{1}{n} S_{n}(\varphi)>0 .
\end{aligned}
$$

The implication $2 \Rightarrow 3$ is a direct consequence of the following Ruelle's inequality: $\max 2\left\{\chi_{\nu}(f), 0\right\} \geq h_{\nu}(f)>0$; see for example [11,15].

It remains to prove the implication $3 \Rightarrow 1$. First, as the proof of [9, Main Theorem] without changes, we have

$$
\limsup _{n \rightarrow \infty}\left(\sup _{I} \frac{1}{n} S_{n}(\varphi)\right) \leq \sup _{\nu \in \mathcal{M}(I, f)} \int_{I} \varphi d \nu .
$$

To prove that $\varphi$ is hyperbolic for $f$, let $\nu_{0}$ be an invariant probability measure maximizing the function $\nu \mapsto \int_{I} \varphi d \nu$. Then for almost every ergodic component $\nu_{0}^{\prime}$ 
of $\nu_{0}$, we have $\int_{I} \varphi d \nu_{0}^{\prime}=\int_{I} \varphi d \nu_{0}$. Thus, the Key Lemma applied to such a $\nu_{0}^{\prime}$, together with Lemma 2.1, implies

$$
P(f, \varphi)>\int_{I} \varphi d \nu_{0}^{\prime}=\int_{I} \varphi d \nu_{0}=\sup _{\nu \in \mathcal{M}(I, f)} \int_{I} \varphi d \nu
$$

Together with (2.1), this implies that $\varphi$ is hyperbolic for $f$ and completes the proof of Theorem 1 .

\section{Proof of the Key Lemma}

In this section, we construct first an "Iterated Multivalued Function System" and then use it to prove our Key Lemma.

3.1. Iterated Multivalued Function Systems. This subsection is devoted to the construction of an "Iterated Multivalued Function System", which is the main ingredient in the proof of the Key Lemma. It is stated as Proposition 3.1, below.

Let $f$ be a map in $\mathscr{A}$. Given a compact and connected subset $B_{0}$ of $I$, a sequence of multivalued functions $\left(\phi_{l}\right)_{l=1}^{+\infty}$ is an Iterated Multivalued Function System (IMFS) generated by $f$ if for every $l$ there is an integer $m_{l} \geq 1$ and a pull-back $W_{l}$ of $B_{0}$ by $f^{m_{l}}$ contained in $B_{0}$ such that

$$
f^{m_{l}}\left(W_{l}\right)=B_{0} \quad \text { and } \quad \phi_{l}=\left(\left.f^{m_{l}}\right|_{W_{l}}\right)^{-1} .
$$

In this case, $\left(m_{l}\right)_{l=1}^{+\infty}$ is the time sequence of $\left(\phi_{l}\right)_{l=1}^{+\infty}$, and $\left(\phi_{l}\right)_{l=1}^{+\infty}$ is defined on $B_{0}$. Note that for each subset $A$ of $B_{0}$ and each $l$, the set $\phi_{l}(A):=f^{-m_{l}}(A) \cap W_{l}$ is non-empty.

Let $\left(\phi_{l}\right)_{l=1}^{+\infty}$ be an IMFS generated by $f$ with time sequence $\left(m_{l}\right)_{l=1}^{+\infty}$, defined on a set $B_{0}$. For each integer $n \geq 1$ put $\Sigma_{n}:=\{1,2, \cdots\}^{n}$ and denote the space of all finite words in the alphabet $\{1,2, \ldots$,$\} by \Sigma^{*}:=\bigcup_{n \geq 1} \Sigma_{n}$. For every integer $k \geq 1$ and $\underline{l}=l_{1} \cdots l_{k}$ in $\Sigma^{*}$, put

$$
|\underline{l}|=k, m_{\underline{l}}=m_{l_{1}}+m_{l_{2}}+\cdots+m_{l_{k}} \text { and } \phi_{\underline{l}}=\phi_{l_{1}} \circ \cdots \circ \phi_{l_{k}} .
$$

Note that for every $x_{0}$ in $B_{0}$ and every pair of distinct words $\underline{l}$ and $\underline{l}^{\prime}$ in $\Sigma^{*}$ satisfying $m_{\underline{l}}=m_{\underline{l^{\prime}}}$, we have the following property:

(*) If the sets $\phi_{\underline{l}}\left(x_{0}\right)$ and $\phi_{\underline{l}^{\prime}}\left(x_{0}\right)$ intersect, then they coincide.

The IMFS $\left(\phi_{l}\right)_{l=1}^{+\infty}$ is free if there is $x_{0}$ in $B_{0}$ such that for every pair of distinct words $\underline{l}$ and $\underline{l}^{\prime}$ in $\Sigma^{*}$ such that $m_{\underline{l}}=m_{l^{\prime}}$, the sets $\phi_{\underline{l}}\left(x_{0}\right)$ and $\phi_{l^{\prime}}\left(x_{0}\right)$ are disjoint.

Proposition 3.1. Let $f$ be an interval map in $\mathscr{A}$ that is topologically exact. Let $\varphi$ : $I \rightarrow \mathbb{R}$ be Hölder continuous, $t \geq 0$ and put $\psi_{t}:=\varphi-t \ln \left|f^{\prime}\right|$. Let $\nu$ be an ergodic invariant probability measure that is not supported on a periodic orbit and that has strictly positive Lyapunov exponent. Then there exists a subset $X$ of $I$ of full measure with respect to $\nu$, such that for every point $x_{0}$ in $X$ the following property holds: There exist $D$ in $(0,+\infty)$, a compact and connected subset $B_{0}$ of $I$ containing $x_{0}$, and a free IMFS $\left(\phi_{l}\right)_{l=1}^{+\infty}$ generated by $f$ with time sequence $\left(m_{l}\right)_{l=1}^{+\infty}$ such that $\left(\phi_{l}\right)_{l=1}^{+\infty}$ is defined on $B_{0}$ and such that for every $l$ and every $y$ in $\phi_{l}\left(B_{0}\right)$ we have

$$
S_{m_{l}}\left(\psi_{t}\right)(y) \geq m_{l} \int_{I} \psi_{t} d \nu-D
$$


The idea of the proof of this proposition is similar to the proof of [9, Proposition 3.1]. Its proof, depending on several lemmas, is given at the end of this subsection.

We proceed first to recall the natural extension of $f$. Let $\mathbb{Z}_{-}$denote the set of all non-positive integers and endow

$$
\mathcal{Z}:=\left\{\left(z_{m}\right)_{m \in \mathbb{Z}_{-}} \in I^{\mathbb{Z}_{-}}: \text {for every } m \in \mathbb{Z}_{-}, f\left(z_{m-1}\right)=z_{m}\right\}
$$

with the product topology. Define $T: \mathcal{Z} \rightarrow \mathcal{Z}$ by

$$
T\left(\left(\cdots, z_{-2}, z_{-1}, z_{0}\right)\right)=\left(\cdots, z_{-2}, z_{-1}, z_{0}, f\left(z_{0}\right)\right)
$$

and $\pi: \mathcal{Z} \rightarrow I$ by $\pi\left(\left(z_{m}\right)_{m \in \mathbb{Z}_{-}}\right)=z_{0}$. Note that $T$ is a bijection, $T^{-1}$ is measurable, $\pi$ is continuous and onto, and $\pi \circ T=f \circ \pi$. If $\nu$ is a Borel probability measure on $I$ that is invariant and ergodic for $f$, then there exists a unique Borel probability measure $\widetilde{\nu}$ on $\mathcal{Z}$ that is invariant and ergodic for $T$ and that satisfies $\pi_{*} \widetilde{\nu}=\nu$; see for example [11, §2.7]. We call $(\mathcal{Z}, T, \widetilde{\nu})$ the natural extension of $(I, f, \nu)$.

The following is a well-known consequence of the pointwise ergodic theorem; see for example [10, Lemma 1.3] for a proof.

Lemma 3.2. Let $(\mathcal{Z}, \mathscr{B}, \widetilde{\nu})$ be a probability space, and let $T: \mathcal{Z} \rightarrow \mathcal{Z}$ be an ergodic measure preserving transformation. Then for each function $\phi: \mathcal{Z} \rightarrow \mathbb{R}$ that is integrable with respect to $\widetilde{\nu}$, there exists a subset $Z$ of $\mathcal{Z}$ such that $\widetilde{\nu}(Z)=1$ and such that for every $\underline{z}$ in $Z$ we have

$$
\limsup _{n \rightarrow \infty} \sum_{i=0}^{n-1}\left(\phi\left(T^{i}(\underline{z})\right)-\int_{\mathcal{Z}} \phi d \widetilde{\nu}\right) \geq 0 .
$$

We need the following lemma which is a version of Ledrappier's unstable manifold theorem; see [5] for the proof.

Lemma 3.3 (Theorem 16, [5]). Let $f$ be an interval map in $\mathscr{A}$. Suppose $\nu$ in $\mathcal{M}(I, f)$ has strictly positive finite Lyapunov exponent. Denote by $(\mathcal{Z}, T, \widetilde{\nu})$ the natural extension of $(I, f, \nu)$. Then there exists a measurable function $\alpha$ on $\mathcal{Z}$ such that $0<\alpha<1 / 2$ almost everywhere with respect to $\widetilde{\nu}$ and such that for $\widetilde{\nu}$-almost every point $y$ in $\mathcal{Z}$ there exists a set $V_{y}$ contained in $\mathcal{Z}$ with the following properties:

1. $y$ is in $V_{y}$ and $\pi\left(V_{y}\right)=B(\pi(y), \alpha(y))$.

2. For each integer $n \geq 0, f^{n}: \pi\left(T^{-n}\left(V_{y}\right)\right) \rightarrow \pi\left(V_{y}\right)$ is diffeomorphic.

3. For each $y^{\prime}$ in $V_{y}$,

$$
\sum_{i=0}^{+\infty}|\log | D f\left(\pi\left(T^{-i}\left(y^{\prime}\right)\right)\right)|-\log | D f\left(\pi\left(T^{-i}\left(y^{\prime}\right)\right)\right)||<\log 2 .
$$

4. For each $\eta>0$ there is a measurable function $\theta$ on $\mathcal{Z}$ with $0<\theta<+\infty$ almost everywhere with respect to $\widetilde{\nu}$ and such that

$$
\frac{1}{\theta(y)} \exp \left(n\left(\chi_{\nu}-\eta\right)\right) \leq\left|D f^{n}\left(\pi\left(F^{-n}(y)\right)\right)\right| \leq \theta(y) \exp \left(n\left(\chi_{\nu}+\eta\right)\right) .
$$

In particular,

$$
\left|\pi\left(T^{-n}\left(V_{y}\right)\right)\right| \leq 2 \theta(y) \exp \left(-n\left(\chi_{\nu}-\eta\right)\right) .
$$

Lemma 3.4. Let $f: I \rightarrow I$ be a map in $\mathscr{A}, \nu$ an invariant ergodic probability measure with strictly positive Lyapunov exponent, $\varphi: I \rightarrow \mathbb{R}$ Hölder continuous, and $t$ in $\mathbb{R}$. Then there exists a subset $X^{\prime}$ of I of full measure with respect to $\nu$, such 
that the following holds. For every point $x$ of $X^{\prime}$ there exist $\rho_{x}>0, D^{\prime}>0$ and a strictly increasing sequence of positive integers $\left(n_{l}\right)_{l=1}^{+\infty}$ such that for every $l \geq 1$ we can choose a point $x_{l}$ in $f^{-n_{l}}(x)$ and a connected component $W_{l}$ of $f^{-n_{l}}\left(B\left(x, \rho_{x}\right)\right)$ containing $x_{l}$ so that:

1. $x_{l+1}$ is in $f^{-\left(n_{l+1}-n_{l}\right)}\left(x_{l}\right)$.

2. Letting $\psi_{t}:=\varphi-t \ln \left|f^{\prime}\right|$, for every point $y$ in $W_{l}$,

$$
S_{n_{l}}\left(\psi_{t}\right)(y) \geq n_{l} \int_{I} \psi_{t} d \nu-D^{\prime}
$$

3. $\lim _{l \rightarrow+\infty}\left|W_{l}\right|=0$.

Proof. Let $(\mathcal{Z}, T, \widetilde{\nu})$ be the natural extension of $(I, f, \nu)$, and note that $\widetilde{\nu}$ is also ergodic with respect to $T^{-1}$. Applying Lemma 3.2 for $T^{-1}$ to the integrable function $\phi=\psi_{t} \circ \pi=\left(\varphi-t \ln \left|f^{\prime}\right|\right) \circ \pi$, we obtain that there exists a subset $Z$ of $\mathcal{Z}$ of full measure with respect to $\widetilde{\nu}$, such that for every point $\left(z_{m}\right)_{m \in \mathbb{Z}_{-}}$in $Z$ we have

$$
\limsup _{n \rightarrow \infty} \sum_{i=0}^{n-1}\left(\psi_{t} \circ \pi\left(T^{-i}\left(\left(z_{m}\right)_{m \in \mathbb{Z}_{-}}\right)\right)-\int_{\mathcal{Z}} \psi_{t} \circ \pi d \widetilde{\nu}\right) \geq 0 .
$$

Taking a subset of $Z$ of full measure with respect to $\widetilde{\nu}$ if necessary, by Lemma 3.3 we can assume that there is a function $\alpha: Z \rightarrow(0,1 / 2)$ such that $Z$ and $\alpha$ satisfy the assertions of Lemma 3.3. Since the set $X^{\prime}:=\pi(Z)$ satisfies $\nu\left(X^{\prime}\right)=$ $\widetilde{\nu}\left(\pi^{-1}(\pi(Z))\right) \geq \widetilde{\nu}(Z)=1$, we have $\nu\left(X^{\prime}\right)=1$.

It remains to verify that $X^{\prime}$ satisfies the desired properties. Fix a point $x$ in $X^{\prime}$ and choose a point $\left(z_{m}\right)_{m \in \mathbb{Z}_{-}}$of $Z$ such that $\pi\left(\left(z_{m}\right)_{m \in \mathbb{Z}_{-}}\right)=x$. Let $V_{\left(z_{m}\right)_{m \in \mathbb{Z}_{-}}}$ be given by Lemma 3.3 for the point $\left(z_{m}\right)_{m \in \mathbb{Z}_{-}}$, and put $\rho_{x}:=\alpha\left(\left(z_{m}\right)_{m \in \mathbb{Z}_{-}}\right)$. Moreover, for each integer $j \geq 1$ put

$$
y_{j}:=\pi\left(T^{-j}\left(\left(z_{m}\right)_{m \in \mathbb{Z}_{-}}\right)\right)=z_{j} \in f^{-j}(x)
$$

and $U_{j}:=\pi\left(F^{-j}\left(V_{\left(z_{m}\right)_{m \in \mathbb{Z}_{-}}}\right)\right)$. By parts 1 and 2 of Lemma 3.3 we know that for every integer $j \geq 1, U_{j}$ is the connected component of $f^{-j}\left(B\left(x, \rho_{x}\right)\right)$ containing $y_{j}$ and $f^{j}: U_{j} \rightarrow B\left(x, \rho_{x}\right)$ is diffeomorphic. On the other hand, by parts 3 and 4 of Lemma 3.3 there exist $C^{\prime}>0$ and $\lambda>1$ such that for every $n \geq 1$ we have $\left|U_{n}\right| \leq C^{\prime} \lambda^{-n}$ and for every pair of points $x, y$ in $U_{n}$,

$$
\frac{1}{2} \leq \frac{\left|\left(f^{n}\right)^{\prime}(x)\right|}{\left|\left(f^{n}\right)^{\prime}(y)\right|} \leq 2 .
$$

Since $\varphi$ is Hölder continuous, we have that there is $\widetilde{C}>1$ such that for every $n \geq 1$ and every pair of points $x, y$ in $U_{n}$,

$$
\left|S_{n}(\varphi)(x)-S_{n}(\varphi)(y)\right| \leq \widetilde{C} .
$$

Fix $D^{\prime \prime}>0$. Then by (3.2) there is a strictly increasing sequence of positive integers $\left(n_{l}\right)_{l=1}^{+\infty}$ such that for every integer $l \geq 1$, we have

$$
\sum_{i=0}^{n_{l}-1} \psi_{t} \circ \pi\left(T^{-i}\left(\left(z_{m}\right)_{m \in \mathbb{Z}_{-}}\right)\right) \geq n_{l} \int_{\mathcal{Z}} \psi_{t} \circ \pi d \widetilde{\nu}-D^{\prime \prime}=n_{l} \int_{I} \psi_{t} d \nu-D^{\prime \prime} .
$$

Therefore, if for each integer $l \geq 1$ we put $x_{l}:=y_{n_{l}}$ and $W_{l}:=U_{n_{l}}$, then parts 1 and 3 are direct consequences of the definitions, and part 2 follows from (3.3), (3.4) and (3.5) with $D^{\prime}=D^{\prime \prime}+\widetilde{C}+t \ln 2$. The proof is completed. 
Lemma 3.5. Let $f$ be an interval map in $\mathscr{A}$ that is topologically exact and $x_{0}$ a interior point of $I$. Then for every open interval $U \subset I$ and every sufficiently large integer $n \geq 1$, there exist two distinct points $y_{1}, y_{2}$ of $f^{-n}\left(x_{0}\right)$ in $U$ such that the following hold:

1. For every $\varepsilon>0$ both of sets $f^{n}\left(B\left(y_{1}, \varepsilon\right)\right)$ and $f^{n}\left(B\left(y_{2}, \varepsilon\right)\right)$ intersect $\left(x_{0},+\infty\right)$.

2. For every $\varepsilon>0$ both of sets $f^{n}\left(B\left(y_{1}, \varepsilon\right)\right)$ and $f^{n}\left(B\left(y_{2}, \varepsilon\right)\right)$ intersect $\left(-\infty, x_{0}\right)$.

Proof. We give the proof of part 1 only, and then the proof can be applied to part 2 without changes. Let $U_{1}$ and $U_{2}$ be two disjoint open subintervals of $U$. Using that $f$ is topologically exact, we know that there is an integer $N \geq 1$ such that for every $n \geq N$ we have $f^{n}\left(U_{1}\right)=I$ and $f^{n}\left(U_{2}\right)=I$. Fix $n \geq N$. Note that the set $f^{-n}\left(x_{0}\right)$ is finite and there are points $z_{1} \in U_{1}$ and $z_{2} \in U_{2}$ such that $f^{n}\left(z_{1}\right)$ and $f^{n}\left(z_{2}\right)$ are in $\left(x_{0},+\infty\right)$. For each $i=1,2$, let $y_{i}$ be a point of $f^{-n}\left(x_{0}\right)$ in $U_{i}$ such that for every $y^{\prime}$ of $f^{-n}\left(x_{0}\right)$ in $U_{i}$ we have $\left|y_{i}-z_{i}\right| \leq\left|y^{\prime}-z_{i}\right|$.

Now let us prove that the lemma holds for such $y_{1}$ and $y_{2}$. Obviously, $y_{1}$ and $y_{2}$ are distinct. To prove that for every $\varepsilon>0$ the set $f^{n}\left(B\left(y_{1}, \varepsilon\right)\right)$ intersects $\left(x_{0},+\infty\right)$, by contradiction, there is $\varepsilon_{0} \in\left(0,\left|y_{1}-z_{1}\right|\right)$ such that $f^{n}\left(B\left(y_{1}, \varepsilon_{0}\right)\right)$ is contained in $\left(-\infty, x_{0}\right]$. It follows that there is a point $z^{\prime}$ of $B\left(y_{1}, \varepsilon_{0}\right) \cap U_{1}$ such that $f^{n}\left(z^{\prime}\right)$ is in $\left(-\infty, x_{0}\right)$ and $\left|z^{\prime}-z_{1}\right|<\left|y_{1}-z_{1}\right|$. Since $f^{n}$ is continuous on $U_{1}$ it follows that there is $y^{\prime \prime}$ in $U_{1}$ such that $\left|y^{\prime \prime}-z_{1}\right|<\left|y_{1}-z_{1}\right|$ and $f^{n}\left(y^{\prime \prime}\right)=x_{0}$. This is a contradiction with our choice of $y$. Using the same method, we can prove for every $\varepsilon>0$ that the set $f^{n}\left(B\left(y_{2}, \varepsilon\right)\right)$ intersects $\left(x_{0},+\infty\right)$. The lemma is proved.

Lemma 3.6 (Lemma 3.2, 9]). For each interval map $f: I \rightarrow I$ in $\mathscr{A}$ there is $\varepsilon>0$ such that the following property holds. Let $J_{0}$ be an interval contained in I satisfying $\left|J_{0}\right| \leq \varepsilon$, let $n \geq 1$ be an integer, and let $J$ be a pull-back of $J_{0}$ by $f^{n}$, such that for each $j$ in $\{1, \ldots, n\}$ the pull-back of $J_{0}$ by $f^{j}$ containing $f^{n-j}(J)$ has length bounded from above by $\varepsilon$. If in addition the closure of $J$ is contained in the interior of $I$, then $f^{n}(\partial J) \subset \partial J_{0}$.

Lemma 3.7 (Lemma A.2, [13]). Let $f: I \rightarrow I$ be an interval map in $\mathscr{A}$ that is topologically exact. Then for every $\kappa>0$ there is $\delta>0$ such that for every $x$ in $I$, every integer $n \geq 1$, and every pull-back $W$ of $B(x, \delta)$ by $f^{n}$, we have $|W|<\kappa$.

Proof of Proposition 3.1. Let $\varepsilon>0$ be the constant given by Lemma 3.6] and let $\delta>0$ be the constant given by Lemma 3.7 for $\kappa=\varepsilon$. Let $X^{\prime}$ be the subset of $I$ given by Lemma 3.4, and let $X$ be the complement in $X^{\prime}$ of the set of periodic points of $f$. Since $\nu$ is ergodic and it is not supported on a periodic orbit, the set $X$ has full measure for $\nu$. Fix a point $x_{0}$ of $X$ that is not an endpoint of $I$.

In part 1 below we define the IMFS, and in part 2 we show it is free and that it satisfies (3.1).

1. Let $\rho_{x_{0}}, D^{\prime},\left(n_{l}\right)_{l=1}^{+\infty},\left(x_{l}\right)_{l=1}^{+\infty}$ and $\left(W_{l}\right)_{l=1}^{+\infty}$ be given by Lemma 3.4 with $x=$ $x_{0}$. Fix $\rho$ in $\left(0, \min \left\{\delta, \rho_{x_{0}}, \operatorname{dist}\left(x_{0}, \partial I\right)\right\}\right)$. Taking a subsequence if necessary, assume $\left(x_{l}\right)_{l=1}^{\infty}$ converges to a point $w_{0}$. Since $f$ is topologically exact, there exist an integer $M \geq 1$ and distinct points $y_{0}$ and $y_{1}$ of $\left(x_{0}-\rho, x_{0}\right)$ such that $f^{M}\left(y_{0}\right)=f^{M}\left(y_{1}\right)=w_{0}$. Let $\rho^{\prime}>0$ be such that the pull-backs $U_{0}$ and $U_{1}$ of $B\left(w_{0}, \rho^{\prime}\right)$ by $f^{M}$ containing $y_{0}$ and $y_{1}$, respectively, are disjoint and contained in $B\left(x_{0}, \rho\right)$. Moreover, by Lemma 3.5 we can choose $M, y_{0}$, and $y_{1}$ so that in addition $U_{0}, U_{1} \subset\left[x_{0}-\rho, x_{0}\right]$, and so that there are infinitely many $l$ for which $x_{l}$ is contained in $f^{M}\left(U_{0}\right)$ and in $f^{M}\left(U_{1}\right)$. 
Using that $\lim _{l \rightarrow+\infty}\left|W_{l}\right|=0$ and taking a subsequence if necessary, assume that for every $l$ we have $n_{l+1}-n_{l} \geq M$, that $\left|W_{l}\right|<\varepsilon$, that the point $x_{l}$ is contained in $f^{M}\left(U_{0}\right)$ and in $f^{M}\left(U_{1}\right)$, and that the pull-back $W_{l}$ of $\overline{B\left(x_{0}, \rho\right)}$ by $f^{n_{l}}$ containing $x_{l}$ is contained in $B\left(w_{0}, \rho^{\prime}\right)$. Interchanging $y_{0}$ and $y_{1}$ and taking a subsequence if necessary, we can also assume that for every $l$ the point $f^{n_{l+1}-n_{l}-M}\left(x_{l+1}\right)$ is not in $U_{0}$. For each $l$ choose a pull-back $W_{l}^{\prime}$ of $W_{l}$ by $f^{M}$ that contains a point $x_{l}^{\prime}$ of $f^{-M}\left(x_{l}\right)$ and that is contained in $U_{0}$.

Note that $W_{l}^{\prime}$ is contained in $U_{0} \subset\left[x_{0}-\rho, x_{0}\right]$, so the closure of $W_{l}^{\prime}$ is contained in the interior of $I$. By Lemma 3.7 and the choice of $\rho$, we know that for every $i$ in $\{0,1, \cdots, n-1\}$ the length of $f^{i}\left(W_{l}^{\prime}\right)$ is less than $\varepsilon$. So by Lemma 3.6 the set $f^{n_{l}+M}\left(\partial W_{l}^{\prime}\right)$ is contained in $\partial B\left(x_{0}, \rho\right)$. Thus, for each $l$ the set $f^{n_{l}+M}\left(W_{l}^{\prime}\right)$ contains either $\left[x_{0}-\rho, x_{0}\right]$ or $\left[x_{0}, x_{0}+\rho\right]$. Suppose first there are infinitely many $l$ such that $f^{n_{l}+M}\left(W_{l}^{\prime}\right)$ contains $\left[x_{0}-\rho, x_{0}\right]$. Taking a subsequence if necessary, assume this holds for every $l$. Then for every $l$ there is a pull-back $W_{l}^{\prime \prime}$ of $\left[x_{0}-\rho, x_{0}\right]$ by $f^{n_{l}+M}$ that is contained in $W_{l}^{\prime}$ and such that $f^{n_{l}+M}\left(W_{l}^{\prime \prime}\right)=\left[x_{0}-\rho, x_{0}\right]$. In this case we put

$$
B_{0}:=\left[x_{0}-\rho, x_{0}\right], M^{\prime}:=M, \text { and } U_{0}^{\prime}:=U_{0},
$$

and note that $W_{l}^{\prime \prime} \subset W_{l}^{\prime} \subset U_{0}^{\prime} \subset\left[x_{0}-\rho, x_{0}\right]=B_{0}$. It remains to consider the case where for each $l$, outside finitely many exceptions, the set $f^{n_{l}+M}\left(W_{l}^{\prime}\right)$ contains $\left[x_{0}, x_{0}+\rho\right]$, but it does not contain $\left[x_{0}-\rho, x_{0}\right]$. Taking a subsequence if necessary, assume this holds for every $l$. Since $f$ is topologically exact, by Lemma 3.5 there is an integer $\widetilde{M} \geq 1$ and a pull-back $U_{0}^{\prime}$ of $U_{0}$ by $f^{\widetilde{M}}$ that is contained in $\left(x_{0}, x_{0}+\rho\right)$, and such that for infinitely many $l$ the point $x_{l}^{\prime}$ is contained in $f^{\widetilde{M}}\left(U_{0}^{\prime}\right)$. Taking a subsequence if necessary, assume that for every $l$ we have $n_{l+1}-n_{l} \geq M+\widetilde{M}$ and that the point $x_{l}^{\prime}$ is contained in $f^{\widetilde{M}}\left(U_{0}^{\prime}\right)$. Since for each $l$ the point $f^{n_{l+1}-n_{l}-M}\left(x_{l+1}\right)$ is not in $U_{0}$, it follows that the point $f^{n_{l+1}-n_{l}-M-\widetilde{M}}\left(x_{l+1}\right)$ is not in $U_{0}^{\prime}$. For each $l$ choose a pull-back $\widetilde{W}_{l}^{\prime}$ of $W_{l}^{\prime}$ by $f^{\widetilde{M}}$ contained in $U_{0}^{\prime}$ and that contains a point of $f^{-\widetilde{M}}\left(x_{l}^{\prime}\right)$. By Lemmas 3.6 and 3.7 again, the set $f^{n_{l}+M+\widetilde{M}}\left(\partial \widetilde{W}_{l}^{\prime}\right)$ is contained in $\partial B\left(x_{0}, \rho\right)$. Since the set $f^{n_{l}+M+\widetilde{M}}\left(\widetilde{W}_{l}^{\prime}\right)$ is contained in $f^{n_{l}+M}\left(W_{l}^{\prime}\right)$ and this last set does not contain $\left[x_{0}-\rho\right.$, $x_{0}$ ], we conclude that $f^{n_{l}+M+\widetilde{M}}$ maps both endpoints of $\widetilde{W}_{l}^{\prime}$ to $x=x_{0}+\rho$. Since by construction $f^{n_{l}+M+\widetilde{M}}\left(\widetilde{W_{l}^{\prime}}\right)$ contains $x=x_{0}$, we conclude that $f^{n_{l}+M+\widetilde{M}}\left(\widetilde{W}_{l}^{\prime}\right)$ contains $\left[x_{0}, x_{0}+\rho\right]$. So there is a pull-back $W_{l}^{\prime \prime}$ of $\left[x_{0}, x_{0}+\rho\right]$ by $f^{n_{l}+M+\widetilde{M}}$ that is contained in $\widetilde{W}_{l}^{\prime}$, and such that $f^{n_{l}+M+\widetilde{M}}\left(W_{l}^{\prime \prime}\right)=\left[x_{0}, x_{0}+\rho\right]$. Note that $W_{l}^{\prime \prime} \subset \widetilde{W}_{l}^{\prime} \subset U_{0}^{\prime} \subset\left(x_{0}, x_{0}+\rho\right)$. In this case we put $B_{0}:=\left[x_{0}, x_{0}+\rho\right]$, and $M^{\prime}:=M+\widetilde{M}$.

Now for each integer $l \geq 1$ we put

$$
\phi_{l}:=\left(\left.f^{n_{l}+M^{\prime}}\right|_{W_{l}^{\prime \prime}}\right)^{-1} .
$$

Then, $\left(\phi_{l}\right)_{l=1}^{+\infty}$ is an IMFS generated by $f$ with time sequence $\left(m_{l}\right)_{l=0}^{+\infty}:=$ $\left(n_{l}+M^{\prime}\right)_{l=0}^{+\infty}$ that is defined on $B_{0}$. Moreover, for each integer $l \geq 1$ we have

$$
n_{l+1}-n_{l} \geq M^{\prime}, W_{l}^{\prime \prime} \subset U_{0}^{\prime} \text {, and } f^{n_{l+1}-n_{l}-M^{\prime}}\left(x_{l+1}\right) \notin U_{0}^{\prime} .
$$

2. To prove that the IMFS $\left(\phi_{l}\right)_{l=1}^{+\infty}$ is free, let $k \geq 1$ and $k^{\prime} \geq 1$ be integers and let

$$
\underline{l}:=l_{1} l_{2} \cdots l_{k} \text { and } \underline{l}^{\prime}:=l_{1}^{\prime} l_{2}^{\prime} \cdots l_{k^{\prime}}^{\prime}
$$


be different words in $\Sigma^{*}$ such that $m_{\underline{l}}=m_{\underline{l^{\prime}}}$. Assume without loss of generality that $l_{k^{\prime}}^{\prime} \geq l_{k}+1$. Note that the set

$$
f^{m_{\underline{l}}-m_{l_{k}}}\left(\phi_{\underline{l}}\left(x_{0}\right)\right)=\phi_{l_{k}}\left(x_{0}\right)
$$

is contained in $W_{l_{k}}^{\prime \prime}$, and therefore in $U_{0}^{\prime}$. On the other hand, we have

$$
m_{l_{k^{\prime}}^{\prime}}-m_{l_{k}}=n_{l_{k^{\prime}}^{\prime}}-n_{l_{k}} \geq n_{l_{k}+1}-n_{l_{k}} \geq M^{\prime},
$$

and therefore the set

$$
\begin{aligned}
f^{m_{\underline{l}}-m_{l_{k}}}\left(\phi_{{\underline{l^{\prime}}}^{\prime}}\left(x_{0}\right)\right)=f^{m_{\underline{l^{\prime}}}-m_{l_{k}}}\left(\phi_{{\underline{l^{\prime}}}^{\prime}}\left(x_{0}\right)\right) & =f^{m_{l_{k^{\prime}}}-m_{l_{k}}}\left(\phi_{l_{k^{\prime}}}\left(x_{0}\right)\right) \\
& =f^{m_{l_{k^{\prime}}^{\prime}}-m_{l_{k}}-M^{\prime}}\left(\left(f^{\left.n_{k^{\prime}}\right|_{W_{l_{k^{\prime}}}}}\right)^{-1}\left(x_{0}\right)\right)
\end{aligned}
$$

contains the point

$$
f^{m_{l_{k^{\prime}}^{\prime}}-m_{l_{k}}-M^{\prime}}\left(x_{l_{k^{\prime}}^{\prime}}\right)=f^{n_{l_{k^{\prime}}}-n_{l_{k}}-M^{\prime}}\left(x_{l_{k^{\prime}}^{\prime}}\right)=f^{n_{l_{k}+1}-n_{l_{k}}-M^{\prime}}\left(x_{l_{k}+1}\right) .
$$

By construction this point is not in $U_{0}^{\prime}$, so we conclude that the sets

$$
f^{m_{\underline{\underline{l}}}-m_{l_{k}}}\left(\phi_{\underline{\underline{l}}}\left(x_{0}\right)\right) \text { and } f^{m_{\underline{\underline{l}}}-m_{l_{k}}}\left(\phi_{{\underline{l^{\prime}}}^{\prime}}\left(x_{0}\right)\right)
$$

are different. This implies that the sets $\phi_{\underline{l}}\left(x_{0}\right)$ and $\phi_{\underline{l}^{\prime}}\left(x_{0}\right)$ are different and by property $(*)$ stated above, that they are disjoint. This completes the proof that the $\operatorname{IMFS}\left(\phi_{l}\right)_{l=1}^{\infty}$ is free.

Finally, let us check inequality (3.1) in the statement of the proposition. Let $C_{1}:=\inf _{I} \varphi-t \sup _{I} \ln \left|f^{\prime}\right|$. Note that $t \geq 0$ and $\sup _{I} \ln \left|f^{\prime}\right|>-\infty$; then $-C_{1}<+\infty$ and $\inf _{I} \psi_{t}=\inf _{I}\left(\varphi-t \ln \left|f^{\prime}\right|\right) \geq C_{1}$. Recall that for every integer $l \geq 1$ and $y$ in $\phi_{l}\left(B_{0}\right)$, the point $f^{M^{\prime}}(y)$ is in $W_{l}$. Thus, by part 2 of Lemma 3.4 we have

$$
\begin{aligned}
S_{m_{l}}\left(\psi_{t}\right)(y)=S_{n_{l}}\left(\psi_{t}\right)\left(f^{M^{\prime}}(y)\right) & +S_{M^{\prime}}\left(\psi_{t}\right)(y) \geq n_{l} \int_{I} \psi_{t} d \nu-D^{\prime}+S_{M^{\prime}}\left(\psi_{t}\right)(y) \\
\geq m_{l} \int_{I} \psi_{t} d \nu & -D^{\prime}-M^{\prime} \int_{I} \psi_{t} d \nu+M^{\prime} \inf _{I} \psi_{t} \\
& \geq m_{l} \int_{I} \psi_{t} d \nu-\left(D^{\prime}+M^{\prime}\left(\int_{I} \psi_{t} d \nu-C_{1}\right)\right) .
\end{aligned}
$$

This proves (3.1) with $D=\max \left\{0, D^{\prime}+M^{\prime}\left(\int_{I} \psi_{t} d \nu-C_{1}\right)\right\}<+\infty$ and completes the proof of the proposition.

3.2. Proof of the Key Lemma. In this subsection we complete the proof of the Key Lemma. The case where the measure $\nu$ is supported on a periodic orbit is different. The proof of the Key Lemma is completed after the following lemma. This lemma is an adaptation of [9, Lemma 4.1], and the proof of the Key Lemma is the same as that of [9, Key Lemma]. We provide those proofs again for the reader's convenience.

Recall that for a differentiable map $f: I \rightarrow I$, a periodic point $p$ of $f$ of period $n$ is hyperbolic repelling if $\left|D f^{n}(p)\right|>1$.

Lemma 3.8. Let $f$ be an interval map in $\mathscr{A}$ that is topologically exact. Then for every Hölder continuous function $\varphi: I \rightarrow \mathbb{R}$ and every hyperbolic repelling periodic point $x_{0}$ of $f$ of period $N$, we have

$$
\limsup _{n \rightarrow \infty} \frac{1}{n} \log \sum_{y \in f^{-n}\left(x_{0}\right)} \exp \left(S_{n}(\varphi)(y)\right)>\frac{1}{N} S_{N}(\varphi)\left(x_{0}\right) .
$$


Proof. The proof is divided into two parts. In part 1 we construct an induced map and in part 2 we show an inequality analogous to (3.6) for the induced map, from which (3.6) follows as a direct consequence.

1. Since $x_{0}$ is hyperbolic repelling, there is $\rho>0$ such that there is a local inverse $\phi$ of $f^{2 N}$ defined on $B\left(x_{0}, \rho\right)$ and fixing $x_{0}$. Reducing $\rho$ if necessary, assume that the closure of $\phi\left(B\left(x_{0}, \rho\right)\right)$ is contained in $B\left(x_{0}, \rho\right)$ and that there is $\theta$ in $(0,1)$ such that $\phi$ contracts distances at least by a factor of $\theta$. Note that $f^{2 N} \circ \phi$ is the identity map on $B\left(x_{0}, \rho\right)$; hence $\phi$ is increasing on $B\left(x_{0}, \rho\right)$ and $f^{2 N}$ is increasing on $\phi\left(B\left(x_{0}, \rho\right)\right)$. Since $f$ is topologically exact, by Lemma 3.5 there is an integer $k^{\prime} \geq 1$ and a point $z^{\prime}$ in $\left(x_{0}, x_{0}+\rho / 2\right)$ such that $f^{2 N k^{\prime}}\left(z^{\prime}\right)=x_{0}$ and such that for every $\varepsilon>0$ the set $f^{2 N k^{\prime}}\left(B\left(z^{\prime}, \varepsilon\right)\right)$ intersects $\left(x_{0}, x_{0}+\rho / 2\right)$. Fix $\varepsilon$ in $\left(0,\left|z^{\prime}-x_{0}\right|\right)$ such that $f^{2 N k^{\prime}}\left(B\left(z^{\prime}, \varepsilon\right)\right) \subset\left(x_{0}, x_{0}+\rho / 2\right)$. Note that the closure of $B\left(z^{\prime}, \varepsilon\right)$ is contained in $\left(x_{0}, x_{0}+\rho / 2\right)$.

Let $W$ be the pull-back of $f^{2 N k^{\prime}}\left(B\left(z^{\prime}, \varepsilon\right)\right) \cap\left[x_{0}, x_{0}+\rho / 2\right)$ by $f^{2 N k^{\prime}}$ containing $z^{\prime}$. Since $f^{2 N k^{\prime}}$, and hence $\varphi^{k^{\prime}}$, is continuous, reducing $\varepsilon$ if necessary, assume that $U_{0}^{\prime}:=\phi^{k^{\prime}}\left(f^{2 N k^{\prime}}(W)\right)$ is disjoint from $\bar{W}$. By our choice of $\phi$, it follows that there is $k_{1} \geq 0$ such that $U_{1}:=\phi^{k_{1}}(W) \subset f^{2 N k^{\prime}}(W)$. Put

$$
k_{0}:=k_{1}+k^{\prime} \text { and } U_{0}:=\phi^{k_{1}}\left(U_{0}^{\prime}\right) .
$$

Then we have $k_{0} \geq 1, U_{0} \cap U_{1}=\emptyset$, and $U_{1} \subset f^{2 N k^{\prime}}(W)$. Since $\phi$ is increasing and contracting and since $f^{2 N k^{\prime}}(W)$ contains $x_{0}$, the set

$$
U_{0}=\phi^{k_{1}}\left(U_{0}^{\prime}\right)=\phi^{k_{0}}\left(f^{2 N k^{\prime}}\left(\overline{B\left(z^{\prime}, \varepsilon\right)}\right)\right)
$$

is contained in $f^{2 N k^{\prime}}(W)$. Finally, note that $f^{2 N k_{0}}\left(U_{1}\right)=f^{2 N k^{\prime}}(W)=f^{2 N k_{0}}\left(U_{0}\right)$. Put

$$
U:=U_{0} \cup U_{1} \text { and } \widehat{f}:=\left.f^{2 N k_{0}}\right|_{U} .
$$

2. Put $\widehat{\varphi}:=\frac{1}{2 N k_{0}} S_{2 N k_{0}}(\varphi)$, for every integer $m \geq 1$ put

$$
\widehat{S}_{m}(\widehat{\varphi}):=\widehat{\varphi}+\widehat{\varphi} \circ \widehat{f}+\cdots+\widehat{\varphi} \circ \widehat{f}^{m-1},
$$

and note that to prove the lemma it suffices to show

$$
\limsup _{m \rightarrow \infty} \frac{1}{m} \log \sum_{y \in \widehat{f}^{-m}\left(x_{0}\right)} \exp \left(\widehat{S}_{m}(\widehat{\varphi})(y)\right)>\widehat{\varphi}\left(x_{0}\right) .
$$

This is equivalent to showing that the radius of convergence of the series

$$
\Xi(s):=\sum_{m=0}^{\infty}\left(\sum_{z \in \widehat{f}^{-m}\left(x_{0}\right)} \exp \left(\widehat{S}_{m}(\widehat{\varphi})(z)\right)\right) s^{m}
$$

is strictly less than $\exp \left(-\widehat{\varphi}\left(x_{0}\right)\right)$. To prove this fact, put $\widehat{K}:=\bigcap_{i=0}^{\infty} \widehat{f}^{-i}(U)$ and observe that $x_{0}$ is contained in this set. Consider the itinerary map

$$
\iota: \widehat{K} \rightarrow\{0,1\}^{\{1,2, \ldots\}}
$$

defined so that for every $i$ in $\{1,2, \ldots\}$ the point $\widehat{f}^{i}(z)$ is in $U_{\iota(z)_{i}}$. Since $\widehat{f}$ maps each of the sets $U_{0}$ and $U_{1}$ onto $f^{2 N k^{\prime}}(W)$ and both of $U_{0}$ and $U_{1}$ are contained 
in this set, for every integer $k \geq 0$ and every sequence $a_{0}, a_{1}, \ldots, a_{k}$ of elements of $\{0,1\}$ there is a point of $\hat{f}^{-(k+1)}\left(x_{0}\right)$ in the set

$$
\widehat{K}\left(a_{0} a_{1} \cdots a_{k}\right):=\left\{z \in \widehat{K} \text { : for every } i \text { in }\{0,1, \cdots, k\} \text { we have } \iota(z)_{i}=a_{i}\right\} .
$$

By our choice of $\phi$ and $U_{0}$, there is a constant $\widehat{C}>0$ such that for every integer $k \geq 1$ and every point $z$ in $\widehat{K}(\underbrace{0 \cdots 0}_{k})$, we have

$$
\widehat{S}_{k}(\widehat{\varphi})(z) \geq k \widehat{\varphi}\left(x_{0}\right)-\widehat{C} .
$$

Taking $\widehat{C}$ larger if necessary, assume that for every point $z$ in $U$ we have

$$
\widehat{\varphi}(z) \geq \widehat{\varphi}\left(x_{0}\right)-\widehat{C} \text {. }
$$

It follows that for every integer $k \geq 0$ and every sequence $a_{0}, a_{1}, \cdots, a_{k}$ of elements of $\{0,1\}$ with $a_{0}=1$ and every point $x$ in $\widehat{K}\left(a_{0} a_{1} \cdots a_{k}\right)$, we have

$$
\widehat{S}_{k+1}(\widehat{\varphi})(x) \geq(k+1) \widehat{\varphi}\left(x_{0}\right)-2\left(a_{0}+a_{1}+\cdots+a_{k}\right) \widehat{C} .
$$

In fact, put $\ell:=a_{0}+\cdots+a_{k}, i_{\ell+1}:=k+1$ and let $0=i_{1}<i_{2}<\cdots<i_{\ell} \leq k$ be all integers $i$ in $\{0,1, \cdots, k\}$ such that $a_{i}=1$. Then by (3.8) and (3.9) for every $j$ in $\{1, \cdots, \ell\}$ we have

$$
\widehat{S}_{i_{j+1}-i_{j}}(\widehat{\varphi})\left(\widehat{f}^{i_{j}}(x)\right) \geq\left(i_{j+1}-i_{j}\right) \widehat{\varphi}\left(x_{0}\right)-2 \widehat{C} .
$$

Summing over $j$ in $\{1,2, \cdots, \ell\}$ we obtain (3.10). Thus, if we put

$$
\Phi(s):=\sum_{k=1}^{\infty} \exp \left(k \widehat{\varphi}\left(x_{0}\right)-2 \widehat{C}\right) s^{k},
$$

then each of the coefficients of

$$
\Upsilon(s):=\Phi(s)+\Phi(s)^{2}+\cdots
$$

is less than or equal to the corresponding coefficient of $\Xi$, and therefore the radius of convergence of $\Xi$ is less than or equal to that of $\Upsilon$. Since clearly $\Phi(s) \rightarrow \infty$ as $s \rightarrow \exp \left(-\widehat{\varphi}\left(x_{0}\right)\right)^{-}$, there is $s_{0}$ in $\left(0, \exp \left(-\widehat{\varphi}\left(x_{0}\right)\right)\right)$ such that $\Phi\left(s_{0}\right) \geq 1$. It follows that the radius of convergence of $\Upsilon$, and hence that of $\Xi$, is less than or equal to $s_{0}$ and therefore it is strictly less than $\exp \left(-\widehat{\varphi}\left(x_{0}\right)\right)$. This completes the proof of (3.7) and of the lemma.

Proof of the Key Lemma. When $\nu$ is supported on a periodic orbit, the desired inequality follows from Lemma 3.8

Now suppose $\nu$ is not supported on a periodic orbit. By Proposition 3.1 with $t=$ 0 , there is $D>0$, a connected and compact subset $B_{0}$ of $I$, and a free $\operatorname{IMFS}\left(\phi_{k}\right)_{k=1}^{\infty}$ generated by $f$ with time sequence $\left(m_{k}\right)_{k=1}^{\infty}$ that is defined on $B_{0}$, and such that for every $k \geq 1$ and every point $y$ in $\phi_{k}\left(B_{0}\right)$ we have

$$
S_{m_{k}}(\varphi)(y) \geq m_{k} \int_{I} \varphi d \nu-D \text {. }
$$

Since the IMFS $\left(\phi_{k}\right)_{k=1}^{\infty}$ is free, there is a point $x_{0}$ of $B_{0}$ such that for every $\underline{l}$ and $\underline{l}^{\prime}$ in $\Sigma^{*}$ such that $m_{\underline{l}}=m_{\underline{l}^{\prime}}$, the sets $\phi_{\underline{l}}\left(x_{0}\right)$ and $\phi_{\underline{l}^{\prime}}\left(x_{0}\right)$ are disjoint. Note that for every integer $k \geq 1$, every $\underline{l}=l_{1} \cdots l_{k}$ in $\Sigma^{*}$, every $y_{0}$ in $\phi_{\underline{l}}\left(x_{0}\right)$, and every $j$ in $\{1, \cdots, k-1\}$, the point

$$
y_{j}:=f^{m_{l_{1}}+m_{l_{2}}+\cdots+m_{l_{j}}}\left(y_{0}\right)
$$


is in $\phi_{m_{l_{j+1}}}\left(B_{0}\right)$. Therefore, by (3.11) we have

$$
\begin{aligned}
S_{m_{\underline{l}}}(\varphi)\left(y_{0}\right)=S_{m_{l_{1}}}(\varphi)\left(y_{0}\right)+S_{m_{l_{2}}}(\varphi)\left(y_{1}\right)+\cdots+S_{m_{l_{k}}}(\varphi)\left(y_{k-1}\right) \\
\quad \geq \sum_{i=1}^{k}\left(m_{l_{i}} \int_{I} \varphi d \nu-D\right)=m_{\underline{l}} \int_{I} \varphi d \nu-k D .
\end{aligned}
$$

This shows that for every $\underline{l}$ in $\Sigma^{*}$ and every $y_{0}$ in $\phi_{\underline{l}}\left(x_{0}\right)$ we have

$$
\exp \left(S_{m_{\underline{l}}}(\varphi)\left(y_{0}\right)\right) \geq \exp \left(m_{\underline{l}} \int_{I} \varphi d \nu\right) \exp (-|\underline{l}| D) .
$$

On the other hand, if for every integer $n \geq 1$ we put

$$
\Xi_{n}:=\bigcup_{\underline{l} \in \Sigma^{*}, m_{\underline{l}}=n} \phi_{\underline{l}}\left(x_{0}\right),
$$

then the radius of convergence of the series

$$
\Xi(s):=\sum_{n=1}^{\infty}\left(\sum_{y \in \Xi_{n}} \exp \left(S_{n}(\varphi)(y)\right)\right) s^{n}
$$

is given by

$$
R:=\left(\limsup _{n \rightarrow \infty}\left(\sum_{y \in \Xi_{n}} \exp \left(S_{n}(\varphi)(y)\right)\right)^{1 / n}\right)^{-1}
$$

and satisfies

$$
\exp \left(-\limsup _{n \rightarrow \infty} \frac{1}{n} \log \sum_{y \in f^{-n}\left(x_{0}\right)} \exp \left(S_{n}(\varphi)(y)\right)\right) \leq R .
$$

Hence, to complete the proof of the Key Lemma it suffices to prove $R<\exp \left(-\int_{I} \varphi d \nu\right)$. Put

$$
\Phi(s):=\sum_{l=1}^{\infty} \exp (-D) \exp \left(m_{l} \int_{I} \varphi d \nu\right) s^{m_{l}} .
$$

By inequality (3.12) and the fact that $\left(\phi_{k}\right)_{k=1}^{\infty}$ is free, each of the coefficients of the series

$$
\Upsilon(s):=\sum_{i=1}^{\infty} \Phi(s)^{i}=\sum_{n=1}^{\infty}\left(\sum_{\underline{l} \in \Sigma^{*}, m_{\underline{l}}=n} \exp \left(m_{\underline{l}} \int_{I} \varphi d \nu\right) \exp (-|\underline{l}| D)\right) s^{n}
$$

does not exceed the corresponding coefficient of the series $\Xi$, so the radius of convergence of $\Xi$ is less than or equal to that of $\Upsilon$. Since clearly $\Phi(s) \rightarrow \infty$ as $s \rightarrow \exp \left(-\int_{I} \varphi d \nu\right)^{-}$, there exists $s_{0}$ in $\left(0, \exp \left(-\int_{I} \varphi d \nu\right)\right)$ such that $\Phi\left(s_{0}\right) \geq 1$. This implies that the radius of convergence of $\Upsilon$, and hence that of $\Xi$, is less than or equal to $s_{0}$ and therefore that $R \leq s_{0}<\exp \left(-\int_{I} \varphi d \nu\right)$. This completes the proof of the Key Lemma.

\section{ACKNOWLEDGment}

The author would like to thank Juan Rivera-Letelier for his useful discussions and help. 


\section{REFERENCES}

[1] Viviane Baladi, Positive transfer operators and decay of correlations, Advanced Series in Nonlinear Dynamics, vol. 16, World Scientific Publishing Co., Inc., River Edge, NJ, 2000. MR.1793194 (2001k:37035)

[2] V. Baladi and G. Keller, Zeta functions and transfer operators for piecewise monotone transformations, Comm. Math. Phys. 127 (1990), no. 3, 459-477. MR.1040891 (91b:58196)

[3] Rufus Bowen, Equilibrium states and the ergodic theory of Anosov diffeomorphisms, Lecture Notes in Mathematics, Vol. 470, Springer-Verlag, Berlin-New York, 1975. MR0442989 (56 \#1364)

[4] Manfred Denker, Gerhard Keller, and Mariusz Urbański, On the uniqueness of equilibrium states for piecewise monotone mappings, Studia Math. 97 (1990), no. 1, 27-36. MR.1074766 (92c:58061)

[5] N. Dobbs, On cusps and flat tops, arXiv:0801.3815v2, 2012.

[6] Franz Hofbauer and Gerhard Keller, Ergodic properties of invariant measures for piecewise monotonic transformations, Math. Z. 180 (1982), no. 1, 119-140, DOI 10.1007/BF01215004. MR656227 (83h:28028)

[7] Irene Inoquio-Renteria and Juan Rivera-Letelier, A characterization of hyperbolic potentials of rational maps, Bull. Braz. Math. Soc. (N.S.) 43 (2012), no. 1, 99-127, DOI 10.1007/s00574012-0007-1. MR.2909925

[8] Gerhard Keller, Generalized bounded variation and applications to piecewise monotonic transformations, Z. Wahrsch. Verw. Gebiete 69 (1985), no. 3, 461-478, DOI 10.1007/BF00532744. MR787608 (86i:28024)

[9] Huaibin Li and Juan Rivera-Letelier, Equilibrium states of weakly hyperbolic one-dimensional maps for Hölder potentials, Comm. Math. Phys. 328 (2014), no. 1, 397-419, DOI 10.1007/s00220-014-1952-x. MR3196990

[10] Feliks Przytycki, Juan Rivera-Letelier, and Stanislav Smirnov, Equality of pressures for rational functions, Ergodic Theory Dynam. Systems 24 (2004), no. 3, 891-914, DOI 10.1017/S0143385703000385. MR2062924(2005e:37103)

[11] Feliks Przytycki and Mariusz Urbański, Conformal fractals: ergodic theory methods, London Mathematical Society Lecture Note Series, vol. 371, Cambridge University Press, Cambridge, 2010. MR2656475(2011g:37002)

[12] J. Rivera-Letelier, Asymptotic expansion of smooth interval maps, arXiv:1204.3071v2, 2012.

[13] J. Rivera-Letelier, On the asymptotic expansion of maps with disconnected Julia set, arXiv:1206.2376v1, 2012.

[14] David Ruelle, A measure associated with axiom-A attractors, Amer. J. Math. 98 (1976), no. 3, 619-654. MR0415683 (54 \#3763)

[15] David Ruelle, An inequality for the entropy of differentiable maps, Bol. Soc. Brasil. Mat. 9 (1978), no. 1, 83-87, DOI 10.1007/BF02584795. MR.516310 (80f:58026)

School of Mathematics and Information Science, Henan University, Kaifeng 475004, PeOple's Republic of ChinA

E-mail address: lihbmath@henu.edu.cn 